\title{
Application of Microfluidics in Bioprocesses
}

\section{Roger C. Lo*}

Department of Chemical Engineering, California State University, Long Beach, Long Beach, CA, USA

\section{What is microfluidics?}

Microfluidics is an interdisciplinary area that involves the study of behaviors of fluids, controlled fluid manipulations, and the design of the devices that can effectively perform such manipulations at microscale (tens to hundreds of micrometers). It has been broadly used to miniaturize analytical methods and chemical/biological processes because of its many advantages, such as significant reduction in analysis time, much lower sample and reagent consumption (in the nanoliter range or less), and enhanced system performance and functionality by integrating different components onto individual devices [1,2]. These applications are usually called micro Total Analysis Systems ( $\mu$ TAS) [3] or lab on a chip (LOC) [4].

One major feature of microfluidic devices is their high surfacearea-to-volume ratio, which leads to enhanced heat and mass transfer, and also interfacial phenomena that are not usually observed at macroscale, such as the domination of surface forces instead of inertial and body forces [5]. The fluid flow within a smooth-walled microchannel is typically in the laminar region with a Reynolds number (Re) less than 100 [6]. The Reynolds number is a dimensionless number representing the ratio of inertial to viscous forces, which indicates the relative importance of these two forces under a given flow condition. It is defined as follows:

$$
\operatorname{Re}=\frac{D V \rho}{\mu}
$$

where $D$ is the hydraulic diameter, $V$ is the characteristic velocity, and $\rho$ is the fluid density. For $R e<2100$, the flow is in the laminar region. Another important feature of microfluidic devices is their capability of integrating multiple steps onto one single device, ranging from sample processing to separation and detection. By mass production, it is possible to develop high-throughput processes using microfluidic devices by parallelization $[7,8]$.

\section{Microfluidic Device Fabrication}

Based on the success of Micro Electro Mechnical Systems (MEMS) and microelectronics industry in the 80 s, microfluidic devices were initially fabricated with glass and silicon using the well-established photolithographic techniques (Figure 1a). Later on, depending on the process parameters, microfluidic devices have been fabricated with different materials, including ceramic, steel, silicone, and Teflon $[9,10]$. Polymers are now often used as construction materials to replace glass and silicon because of their lower cost and simpler fabrication process without the need for nasty chemicals. Particularly for biological applications, Poly Dimethyl Siloxane (PDMS) is the most popular material in microfluidic device fabrication because of its many advantages, such as optical transparency, biocompatibility, elasticity, and simple fabrication process ("soft lithography") [1113] (Figure 1b). More information on PDMS microfluidic devices is available in several reviews and the references cited therein [14-16].

\section{Application in Bioprocesses}

Bioprocesses have severed as an important means to support survival and fulfill different needs for thousands of years in the human history. Early bioprocesses (ca. 4000 BC) employed natural microorganisms, such as yeasts and fungi, to produce different products, including bread, beer, and cheese. The first purification step was ethanol distillation carried out around 2000 BC [17,18]. With advances in science and engineering, bioprocesses increases in both production scale and complexity with integrated processing steps. In addition to nature microorganisms, modern bioprocesses also use other agents, e.g. enzymes and cells from plants, insects, and animals, to produce various products, including organic acids, antibiotics, and therapeutic compounds [19]. Bioprocesses are developed by combining different basic steps (or "unit operations", introduced by Arthur D. Little in 1915), such as fermentation, filtration and drying. For decades, scaling up from benchtop via a pilot plant to a full blown one has been the standard practice of the development of industrialscale bioprocesses. However, it is faced with challenges from the more stringent requirements, such as size and cost reductions in equipment, lower energy consumption and waste emission, and a safer operation

a)

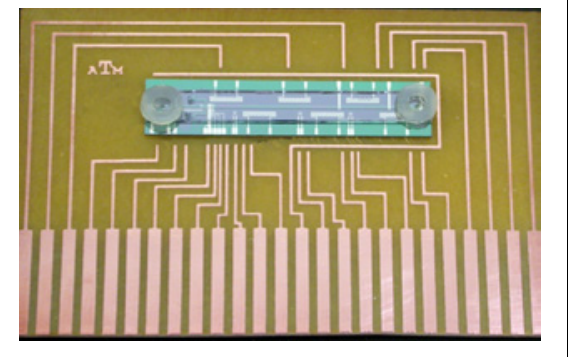

b)

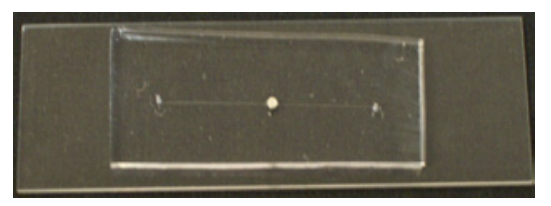

Figure 1: Microfluidic devices for biological applications. a) A device fabricated with glass and silicon using photolithography for microchip DNA gel electrophoresis [29]. b) A device fabricated with glass and PDMS using soft lithography for on-chip enzymatic reactions with derivatized magnetic microbeads [30].

*Corresponding author: Roger C. Lo, Department of Chemical Engineering, California State University, Long Beach, Long Beach, CA 90840, USA, Phone: (562) 985-1508; Email: roger.lo@csulb.edu

Received November 26, 2012; Accepted November 26, 2012; Published December 03, 2012

Citation: Roger C. Lo (2012) Application of Microfluidics in Bioprocesses. J Bioprocess Biotech 2:e109 doi: 10.4172/2155-9821.1000e109

Copyright: (c) 2012 Roger C. Lo. This is an open-access article distributed under the terms of the Creative Commons Attribution License, which permits unrestricted use, distribution, and reproduction in any medium, provided the original author and source are credited. 
environment, due to the new trend of using sustainable production schemes in bioprocesses. To address these challenges, an approach called "process intensification" has been used to improve bioprocesses. It focuses on developing new equipment and methods that leads to more cost-effective and sustainable bioprocesses [20,21].

Since its debut in the 90s [22-24], microfluidics has made significant progress through the piling up research results. It has found many biological applications, such as gene/protein manipulation and analysis, cell-based systems, biosensors, and drug discovery and delivery $[25,26]$. Microfluidic devices have recently come into attention as a powerful tool for bioprocess intensification because of their low fabrication costs and reagent consumption, small form factors for safe operation in a controlled environment, and capability of integrate multiple basic steps onto one chip. A lot of work has been directed to the development of microreactors for enzymatic reactions (e.g. hydrolysis, esterification, oxidation/reduction, and polymerization). For downstream processing, microfluidic devices have been used to develop systems for separation of cells and purification of therapeutic compounds. The results so far are very promising for miniaturized bioprocesses. More information on the miniaturization of bioprocesses can be found in the literature $[27,28]$.

\section{The Outlook}

For the past two decades, microfluidics has made great strides and has gradually moved from pure research projects to commercialized products, such as Agilent Technologies' 2100 Bioanalyzer for biomolecule analysis, Caliper Life Sciences' LabChip systems for biomolecule analysis and drug discovery, and FutureChemistry's microreactor systems for process optimization. Although microfluidic systems are still not the dominating ones in bioprocesses, their future is very promising as indicated in reported research results. Along with advances in materials and fabrication processes, new discoveries of fluid behaviors at microscale might lead to new reaction mechanisms that are not possible on conventional macroscale systems.

Microfluidic systems are also potential for industry-scale bioprocesses because of their capability of parallelization. The throughput of such systems can be significantly enhanced by increasing the number of optimized microreactors ("scale out") instead of the conventional scale-up process that is more expensive and carries more uncertainties. Based on reports in the literature and commercial products currently available, it is foreseeable that there will be cost effective, "plug and play" microfluidic systems with customizable reaction modules for bioprocesses. The user will be able to customize his or her reactions in the desired bioprocess by switching different microreactors, just like what we do today to change our computer configuration by swapping plug and play components.

\section{References}

1. Burns MA, Johnson BN, Brahmasandra SN, Handique K, Webster JR, et al (1998) An Integrated Nanoliter DNA Analysis Device. Science 282: 484-487.

2. Thorsen T, MaerkI SJ, Quake SR (2002) Microfluidic Large-Scale Integration. Science 298: 580-584

3. West J, Becker M, Tombrink S, Manz A (2008) Micro total analysis systems: latest achievements. Anal Chem 80: 4403-4419.

4. Brivio M, Verboom W, Reinhoudt DN (2006) Miniaturized continuous flow reaction vessels: influence on chemical reactions. Lab Chip 6: 329-344.

5. Pennathur S, Meinhart CD, Soh HT (2008) How to exploit the features of microfluidics technology. Lab Chip 8: 20-22.

6. Hetsroni G, Mosyak A, Pogrebnyak E, Yarin LP (2005) Fluid flow in microchannels. Int J Heat Mass Tran 48: 1982-1998.
7. Dittrich PS, Manz A (2006) Lab-on-a-chip: microfluidics in drug discovery Nat Rev Drug Discov 5: 210-218.

8. Melin J, Quake SR (2007) Microfluidic large-scale integration: the evolution of design rules for biological automation. Annu Rev Biophys Biomol Struct 36: $213-231$

9. Goodell JR, McMullen JP, Zaborenko N, Maloney JR, Ho C-X, et al (2009) Development of an Automated Microfluidic Reaction Platform for Multidimensional Screening: Reaction Discovery Employing Bicyclo [3.2.1] octanoid Scaffolds. J Org Chem 74: 6169-6180.

10. Ren K, Dai W, Zhou J, Su J, Wu H (2011) Whole-Teflon microfluidic chips. Proc Natl Acad Sci U S A 108: 8162-8166.

11. Xia Y, Whitesides GM (1998) Soft Lithography. Annu Rev Mater Sci 28: 153 184

12. Whitesides GM, Ostuni E, Takayama S, Jiang X, Ingber DE (2001) Soft Lithography in Biology and Biochemistry. Annu Rev Biomed Eng 3: 335-373.

13. Friend J, Yeo L (2010) Fabrication of microfluidic devices using polydimethylsiloxane. Biomicrofluidics 4 .

14. McDonald JC, Duffy DC, Anderson JR, Chiu DT, Wu H, et al. (2000) Fabrication of microfluidic systems in poly (dimethylsiloxane). Electrophoresis 21: 27-40.

15. McDonald JC, Whitesides GM (2002) Poly (dimethylsiloxane) as a material for fabricating microfluidic devices. Acc Chem Res 35: 491-499.

16. Sia SK, Whitesides GM (2003) Microfluidic devices fabricated in poly(dimethylsiloxane) for biological studies. Electrophoresis 24: 3563-3576.

17. Hulse JH (2004) Biotechnologies: past history, present state and future prospects. Trends Food Sci Tech 15: 3-18.

18. Wilbey RA, Scott JE, Robinson RK (1998) Cheesemaking Practice. (3rd Edn) Springer.

19. Heinzle E, Biwer AP, Cooney CL (2007) Development of Sustainable Bioprocesses: Modeling and Assessment. Wiley.

20. Stankiewicz AI, Moulijn JA (2000) Process intensification: transforming chemical engineering. Chem Eng Prog 96: 22-34

21. Lutze P, Gani R, Woodley JM (2010) Process intensification: A perspective on process synthesis. Chemical Engineering and Processing: Process Intensification 49: 547-558

22. Manz A, Graber N, Widmer HM (1990) Miniaturized total chemical analysis systems: A novel concept for chemical sensing. Sensor Actuat B-Chem 1 : 244-248.

23. Manz A, Harrison DJ, Verpoorte EMJ, Fettinger JC, Lüdi H, et al. (1991) Miniaturization of Chemical Analysis Systems-A Look into Next Century's Technology or Just a Fashionable Craze? CHIMIA International Journal for Chemistry 45: 103-105.

24. Harrison DJ, Glavina PG, Manz A (1993) Towards miniaturized electrophoresis and chemical analysis systems on silicon: an alternative to chemical sensors. Sensor Actuat B: Chem 10: 107-116.

25. El-Ali J, Sorger PK, Jensen KF (2006) Cells on chips. Nature 442: 403-411.

26. Yeo LY, Chang HC, Chan PP, Friend JR (2011) Microfluidic devices for bioapplications. Small 7: 12-48.

27. Asanomi Y, Yamaguchi H, Miyazaki M, Maeda H (2011) Enzyme-immobilized microfluidic process reactors. Molecules 16: 6041-6059.

28. Marques MPC, Fernandes P (2011) Microfluidic Devices: Useful Tools for Bioprocess Intensification. Molecules 16: 8368-8401.

29. Lo RC, Ugaz VM (2008) Microchip DNA electrophoresis with automated whole-gel scanning detection. Lab Chip 8: 2135-2145

30. Liu X, Lo RC, Gomez FA (2009) Fabrication of a microfluidic enzyme reactor utilizing magnetic beads. Electrophoresis 30: 2129-2133. 